\section{Wild variability}

Dolph Schluter

Avian Genetics: A Population and Ecological Approach. Edited by F. Cooke and P.A. Buckley. Academic: 1987. Pp. 488. $£ 40, \$ 72$.

RESEARCH on birds has had more influence on modern evolutionary theory than has study of most other taxa. Concepts as fundamental as clinal variation and the biological species were formulated with Aves in mind. Yet, although their systematics, behaviour and ecology have been extensively investigated, genetic processes in wild birds are still poorly understood.

This volume is the first to summarize what is known of avian population genetics. It contains a diverse collection of papers, written by a variety of researchers, but the material is reasonably well organized. Coherence is aided by the planning of the editors, whose overall intention was to characterize the different types of genetic variation found within species, and the demographic and ecological forces which shape it. Within the confines of this theme, we are treated to an excellent sample of approaches and questions in avian studies. Not all aspects of population genetics are included (for example species formation is hardly touched upon), but the book is a good beginning.

A particular strength is the attention paid to the many problems that remain. A wealth of questions is raised in virtually all the chapters, and many of these are highlighted by the editors in later summaries. The final chapter by Buckley is especially helpful in this regard, synthesizing advances and approaches to date, and pointing the way to the future. The result is a guide book for studies in avian genetics that will be useful to a fairly broad readership.

On the negative side, an excessive amount of the volume is devoted to basic methodology. This is especially true in the first section, which reviews the prevalence of many types of genetic variation in birds (Mendelian and quantitative traits, chromosomes, allozymes and DNA sequences). Several chapters would have benefited from briefer summaries of general technique, and greater discussion of the results of applications to work on birds. More frequent comparison with other taxa would also have been useful. A general conclusion to be drawn from this section is that birds indeed show genetic variation at all levels, but its significance is only dimly grasped and much remains to be catalogued.

The second part of the book deals with population processes moulding genetic that have involved genetic studies. The players are familiar (great tit, house sparrow, snow goose and Arctic skua), though much recent work is described. The most interesting papers are those which manage to integrate genetic studies with other objectives in systematics, behaviour or population dynamics. A good example is that on the snow goose, by Cooke. Here, understanding of the elementary genetic basis of a plumage polymorphism has led to a detailed study of evolutionary processes on a continental scale.

The book is compelling reading because of the large amount of information reviewed, and the outline of many of the genetic and evolutionary problems which remain. In addition, one gains a better appreciation of both the benefits of genetic studies, and of birds as first-class subjects for research. tions into natural selection in the field (here reviewed by Price and Boag).

The concluding section enlarges upon the evolutionary theme of the previous chapters. Included are reviews from an eclectic assortment of research projects

\section{Twinkle, twinkle}

\section{Robert F. Garrison}

The Classification of Stars. By Carlos Jaschek and Mercedes Jaschek. Cambridge University Press: 1987. Pp.413. $£ 45, \$ 79.50$.

Classification is a continuing activity for astronomers; we're not likely to run out of stars. Although it might be nice to have a detailed study of every star, it is far more practical to classify as many stars in as many ways as we can, and then to study the prototypes in detail. Our understanding of the nature of the Universe is improved by the development of better classification techniques. Of course, as fainter stars are surveyed in greater detail with better equipment, new categories present themselves, so the task of astronomical classification is never complete.

There is no comprehensive, booklength survey of the entire field of stellar classification, so the new volume by Carlos and Mercedes Jaschek fills a void. The authors have given us a wealth of information, which is accurate for the most part, and the list of references, though selective, is invaluable as a starting point for the study of any particular type of star. The level is that of the advanced undergraduate or beginning postgraduate student.

There, are, however, a disturbing number of errors. One can forgive some mistakes of grammar, syntax, spelling and typography, but in this case the error level is about one per page, which is very much
Dolph Schluter is Assistant Professor and NSERC University Research Fellow in the Department of Zoology, University of British Columbia, Vancouver, British Columbia V6T 2A9, Canada.

higher than it should be. Most of these slips are just a nuisance and should have been caught by the copy editor, but some are more serious because they are subtle and change the scientific meaning of the passage concerned. The book in general is quite readable, but there is on occasion an irritating awkwardness in the choice of words. For example, "incertitude" is used instead of "uncertainty". While incertitude may well be correct, it is uncommon and (to me at least) appears stilted.

The idea of using tracings to illustrate spectra is a good one. Although photographic spectra might be easier to use in their original form, it is difficult to reproduce them without increasing the contrast and thus losing lines. However, it would have been better if the tracings had been from higher signal-to-noise digital spectra and if the spectra had been smoothed or broken into smaller segments. In some cases, the lines are hard to see, even for an expert.

The Jascheks have made a good attempt at organization of the field and its literature. Much of what I would like to see in such a book is there, though of course not always in the way I would like to see it. In a compilation volume, there is inevitably an author selection-effect, but that is the prerogative of the authors concerned and is one of the reasons for going to the trouble of writing a book. In any case, a student reading this volume would learn a great deal about stellar astronomy. I am using it, among others, for my course in stellar spectroscopy this term.

Robert F. Garrison is Professor of Astronomy at the University of Toronto, Toronto, Ontario MSS 1A7, Canada. 